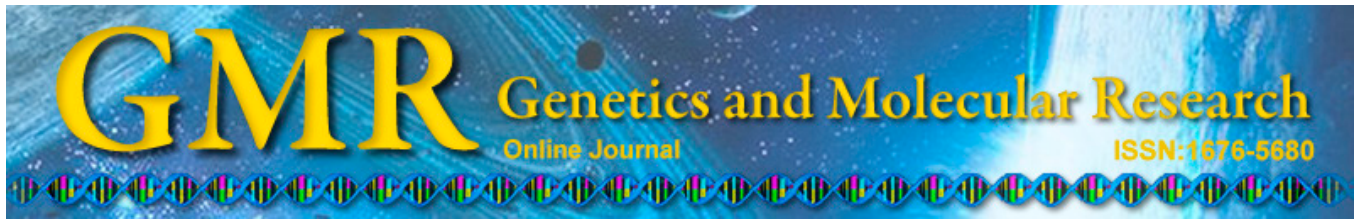

\title{
Study on the ERCC1 gene polymorphism response to chemotherapy and prognosis of gastric cancer
}

\author{
L. Liu ${ }^{1}$, C.H. Li ${ }^{2}$, T.F. Jin ${ }^{2}$ and D.Y. Xu \\ ${ }^{1}$ Department of Pathology, Affiliated Hospital of Yanbian University, \\ Yanji, China \\ ${ }^{2}$ Department of Gastroenterology and Hepatology, \\ Affiliated Hospital of Yanbian University, Yanji, China \\ ${ }^{3}$ Department of Pathology, Center of Morphological Experiment, \\ Medical College of Yanbian University, Yanji, China
}

Corresponding author: D.Y. Xu

E-mail: dongyuanx_ybu@126.com

Genet. Mol. Res. 13 (4): 8722-8728 (2014)

Received September 27, 2013

Accepted March 29, 2014

Published October 27, 2014

DOI http://dx.doi.org/10.4238/2014.October.27.13

ABSTRACT. We conducted a cohort study to investigate the role
of 2 single-nucleotide polymorphisms of the excision repair cross-
complimentary group 1 (ERCC1) gene polymorphism in response to
chemotherapy and clinical outcomes of gastric cancer. A total of 231
patients with newly diagnosed and histopathologically confirmed
primary gastric cancer participated in the study. ERCC1 rs 11615 and
rs3212986 were genotyped. Individuals with the ERCC1 rs 11615 TT
genotype and the T allele showed a significant poorer response to
chemotherapy compared to the wild-type genotype. Patients carrying
the rs 11615 TT genotype ( 22.8 months) and the T allele ( 24.2 months)
showed a significantly shorter median survival time when compared with
the GG genotype ( 33.7 months). Cox proportional hazard regression
analysis showed that adjusted hazard ratios of overall survival in those
carrying the rs 11615 TT genotype and the T allele were 2.79 (1.15-7.26) 
and 1.84 (1.19-2.87) when using the wild-type genotype as a reference variable. In conclusion, this study reports that the ERCC1 rs11615 TT polymorphism can be used as a prognostic marker to determine the clinical outcome of gastric cancer patients treated with 5-fluorouracilbased chemotherapy.

Key words: Excision repair cross-complimentary group 1;

5-Fluorouracil; Chemotherapy response; Gastric cancer; Survival time

\section{INTRODUCTION}

Gastric cancer is the second most common cancer in the Asian population, with approximately half of all cases in China (Ferlay et al., 2010). Surgery is the main treatment for early-stage disease, but most of these patients have advanced cancer or have local or distant recurrence by the time of diagnosis (MacDonald, 2004). Therefore, neoadjuvant chemotherapy followed by surgery has become a main treatment method for prolonging the survival of these patients. 5-Fluorouracil (5-FU) is the main chemotherapeutic agent used to treat gastric cancer, and combination chemotherapy with 5-FU presents an improved clinical outcome (Wöhrer et al., 2004). However, patients show different responses to chemotherapy and different severity or drug-related adverse events even at the same clinical stages. Therefore, genetic variations in drug-metabolizing enzymes may influence responses to chemotherapy and the clinical outcomes of patients.

The nucleotide excision repair (NER) pathway is the most versatile DNA repair mechanism and is responsible for removing a wide variety of DNA lesions (Friedberg, 2001). Excision repair cross-complimentary group 1 (ERCC1) is a major component of the NER pathway, and plays an important role in repairing intrastrand crosslinks in DNA (Zamble et al., 1996). ERCC1 is located on chromosome 19q13.32, interacts with other NER proteins, and guides the 5'-incision activity of the NER pathway (Volker et al., 2001). Previous studies reported that the ERCC1 polymorphism is correlated with increased chemotherapeutic sensitivity and may be a prognostic marker for various cancers treated with chemotherapy (Mlak et al., 2013; Moxley et al., 2013; Rumiato et al., 2013). The association between the ERCC1 polymorphism and gastric cancer has been reported in several studies (Huang et al., 2008; Liang et al., 2010; Yin et al., 2011). However, the results are conflicting. Therefore, we conducted a cohort study to investigate the role of 2 common single-nucleotide polymorphisms (SNPs) of the ERCC1 gene polymorphism in response to chemotherapy and clinical outcomes of gastric cancer.

\section{MATERIAL AND METHODS}

\section{Patient eligibility}

We recruited 256 patients who were newly diagnosed and histologically confirmed to have primary gastric cancer at the Affiliated Hospital of Yanbian University between January 2008 and April 2009. Patients who were not newly diagnosed, who had inadequate organ function, and who were pregnant or breast-feeding were excluded from this study. All patients were interviewed to determine smoking and drinking status. Smoking status was divided into 2 groups. Never smokers were defined as subjects who had smoked less than 100 cigarettes 
in their lifetime, while the remaining subjects were considered as ever smokers. Drinking status was divided into 2 groups: ever drinkers and never drinkers. Ever drinkers were defined as individuals who had drunk alcoholic beverages at least once per week for more than 1 year, while all others were regarded as never drinkers. All patients were asked to provide $5 \mathrm{~mL}$ whole blood for genetic testing. Informed consent was obtained from all patients, and the protocol of this study was approved by the Ethics Committee of the Affiliated Hospital of Yanbian University.

\section{Chemotherapy}

A total of 361 patients were treated with fluorouracil-based chemotherapy. The response to treatment was evaluated according to the World Health Organization criteria (Miller et al., 1981). A complete response was defined as the disappearance of tumor markers for at least 2 weeks. Partial remission was regarded as a reduction of more than $30 \%$ in uni-dimensional tumor size and no appearance or progression of lesions. Progressive disease was defined as the appearance of any new lesions and a $20 \%$ increase in the lesions. The remaining patients were defined as having stable disease. The primary endpoint was overall survival (OS), which was calculated at the date of diagnosis until the date of death from any cause or last follow-up.

\section{Genotyping}

Two potentially common ERCC1 SNPs were selected, including rs11615 and rs3212986. Extraction of genomic DNA from blood samples was conducted using a Qiagen Blood DNA Mini Kit (Qiagen, Hilden, Germany). rs11615 and rs3212986 were genotyped using Taqman assays and the ABI Prism 7911HT Sequence Detection System (Applied Biosystems, Foster City, CA, USA). Primer and probes were designed using the Sequenom Assay Design 3.1 software (Sequenom ${ }^{\circledR}$, San Diego, CA, USA). PCR for rs11615 was performed using the primers forward: 5'-GGATCAGGGACTGTCCAGGGTT-3' and reverse: 5'-CGGGAATTACGTCGCCAAA-3'; PCR for rs3212986 was performed using the primers forward: 5'-TGAGCCAATTCAGCCACTAGAG-3' and reverse: 5'-CTTTAGTTCCTCAGTT TCCCG-3'. The reaction conditions were the same as those in a previous study (Yang et al., 2013). For quality control, we randomly selected $10 \%$ of the samples for repeated genotyping, and the results showed $100 \%$ concordant.

\section{Statistical analysis}

All analyses were conducted using the SPSS version 11.0 statistical software (SPSS, Chicago, IL, USA). The correlation between polymorphisms in ERCC1 rs11615 and rs3212986 and the response to chemotherapy was assessed using logistic regression analysis with odds ratios (ORs) and $95 \%$ confidence intervals $(95 \% \mathrm{CI})$. The association between polymorphisms in ERCC1 rs11615 and rs3212986 and OS was estimated by hazard ratios (HR) and its 95\%CI by multivariate Cox proportional hazard regression analysis. Survival distributions were estimated using the Kaplan-Meier method. All P values were two-sided and $<0.5$ was considered to be statistically significant. 


\section{RESULTS}

Of the 256 patients recruited for this study, 231 met the eligible criteria and agreed to participate, with a participation rate of $90.23 \%$. Of the 231 gastric cancer patients, $152(65.80 \%)$ were males and $79(34.20 \%)$ were females (Table 1). The average age was $55.4 \pm 9.2$ years (range, 24-79 years). Among the 231 patients, $124(53.68 \%)$ and 107 (46.32\%) patients were intestinal and diffuse type, respectively. Additionally, 31 (13.42\%), $36(15.58 \%)$, and $164(71 \%)$ patients showed well, moderate and poor differentiation, respectively. A total of 135 (58.44\%) patients were at ECOG $\leq 1$. There were $139(60.17 \%)$ patients at stage III-IV.

\begin{tabular}{|c|c|c|}
\hline Variables & Patients $(\mathrm{N}=231)$ & $\%$ \\
\hline Mean age (years) & $55.4 \pm 9.2$ & \\
\hline$<55$ & 109 & 47.19 \\
\hline$\geq 55$ & 122 & 52.81 \\
\hline \multicolumn{3}{|l|}{ Gender } \\
\hline Male & 152 & 65.80 \\
\hline Female & 79 & 34.20 \\
\hline \multicolumn{3}{|l|}{ Smoking status } \\
\hline Never & 146 & 63.20 \\
\hline Ever & 85 & 36.80 \\
\hline \multicolumn{3}{|l|}{ Alcohol status } \\
\hline Never & 131 & 56.71 \\
\hline Ever & 100 & 43.29 \\
\hline \multicolumn{3}{|l|}{ Histological type } \\
\hline Intestinal & 124 & 53.68 \\
\hline Diffuse & 107 & 46.32 \\
\hline \multicolumn{3}{|l|}{ Differentiation } \\
\hline Well & 31 & 13.42 \\
\hline Moderate & 36 & 15.58 \\
\hline Poor & 164 & 71.00 \\
\hline \multicolumn{3}{|c|}{ Performance status (ECOG) } \\
\hline$\leq 1$ & 135 & 58.44 \\
\hline$=2$ & 96 & 41.56 \\
\hline \multicolumn{3}{|l|}{ Stage } \\
\hline I-II & 92 & 39.83 \\
\hline III/IV & 139 & 60.17 \\
\hline
\end{tabular}

Among the remaining 231 patients, 102 patients showed a response to chemotherapy (overall chemotherapy response rate, 44.16\%) (Table 2). A statistically significant association was observed in the polymorphism of ERCC1 1 rs 11615 between responders and non-responders $\left(\chi^{2}=8.43, \mathrm{P}=0.015\right)$. Individuals with the ERCC1 rs11615 TT genotype and the T allele showed a significant poor response to chemotherapy compared to the wild-type genotype, with OR $(95 \% \mathrm{CI})$ of $0.34(0.15-0.77)$ and $0.53(0.36-0.80)$, respectively.

\section{Genotypes and overall survival}

All patients were followed up from the time of diagnosis until the end of January 2012. The median follow-up time was 27.5 months (from 1-60 months) (Table 3). A total of 157 patients (67.97\%) died from gastric cancer during the follow-up period. 


\begin{tabular}{|c|c|c|c|c|c|c|c|c|c|}
\hline SNP & & $\begin{array}{l}\text { Total frequencies } \\
\qquad(\mathrm{N}=231)\end{array}$ & $\%$ & $\begin{array}{l}\text { Responders } \\
(\mathrm{N}=102)\end{array}$ & $\%$ & $\begin{array}{l}\text { Non-responders } \\
\quad(\mathrm{N}=129)\end{array}$ & $\%$ & OR $(95 \% \mathrm{CI})$ & $P$ value \\
\hline \multirow[t]{5}{*}{ rs11615 } & $\mathrm{CC}$ & 99 & 42.86 & 53 & 51.96 & 46 & 35.66 & 1.0 (Ref.) & - \\
\hline & $\mathrm{CT}$ & 86 & 37.23 & 36 & 35.29 & 50 & 38.76 & $0.62(0.33-1.17)$ & 0.11 \\
\hline & $\mathrm{TT}$ & 46 & 19.91 & 13 & 12.75 & 33 & 25.58 & $0.34(0.15-0.77)$ & 0.005 \\
\hline & $\mathrm{C}$ allele & 284 & 61.47 & 142 & 69.61 & 142 & 55.04 & 1.0 (Ref.) & 0.001 \\
\hline & $\mathrm{T}$ allele & 178 & 38.53 & 62 & 30.39 & 116 & 44.96 & $0.53(0.36-0.80)$ & \\
\hline \multirow[t]{5}{*}{ rs3212986 } & $\mathrm{CC}$ & 110 & 47.62 & 51 & 50.00 & 59 & 45.74 & 1.0 (Ref.) & \\
\hline & $\mathrm{CA}$ & 85 & 36.80 & 38 & 37.25 & 47 & 36.43 & $0.94(0.51-1.72)$ & 0.82 \\
\hline & $\mathrm{AA}$ & 36 & 15.58 & 13 & 12.75 & 23 & 17.83 & $0.65(0.28-1.51)$ & 0.28 \\
\hline & $\mathrm{C}$ allele & 305 & 66.02 & 140 & 68.63 & 165 & 63.95 & 1.0 (Ref.) & \\
\hline & A allele & 157 & 33.98 & 64 & 31.37 & 93 & 36.05 & $0.81(0.54-1.22)$ & 0.29 \\
\hline
\end{tabular}

Table 3. Univariate analysis of ERCC1 in relation to overall survival of gastric cancer.

\begin{tabular}{|c|c|c|c|c|c|c|c|c|c|}
\hline Gene & & $\begin{array}{c}\text { Cases } \\
(\mathrm{N}=231)\end{array}$ & $\%$ & $\begin{array}{l}\text { Events } \\
(\mathrm{N}=157)\end{array}$ & $\%$ & $\begin{array}{l}\text { Overall survival } \\
\text { Median (months) }\end{array}$ & Log-rank & $\mathrm{HR}(95 \% \mathrm{CI})$ & $P$ value \\
\hline \multirow[t]{5}{*}{ rs11615 } & $\mathrm{CC}$ & 99 & 42.86 & 60 & 38.22 & 33.7 & & 1.0 (Ref.) & - \\
\hline & $\mathrm{CT}$ & 86 & 37.23 & 61 & 38.85 & 26.9 & & $1.65(0.86-3.21)$ & 0.11 \\
\hline & $\mathrm{TT}$ & 46 & 19.91 & 36 & 22.93 & 22.8 & 0.012 & $2.79(1.15-7.26)$ & 0.014 \\
\hline & $\mathrm{C}$ allele & 284 & 61.47 & 181 & 57.64 & 34.9 & & 1.0 (Ref.) & - \\
\hline & $\mathrm{T}$ allele & 178 & 38.53 & 133 & 42.36 & 24.2 & 0.036 & $1.84(1.19-2.87)$ & 0.004 \\
\hline \multirow{5}{*}{ rs3212986 } & $\mathrm{CC}$ & 110 & 47.62 & 71 & 45.22 & 28.3 & & 1.0 (Ref.) & - \\
\hline & $\mathrm{CA}$ & 85 & 36.80 & 59 & 37.58 & 27.1 & & $1.25(0.65-2.39)$ & 0.47 \\
\hline & $\mathrm{AA}$ & 36 & 15.58 & 27 & 17.20 & 26.9 & 0.37 & $1.65(0.67-4.38)$ & 0.25 \\
\hline & $\mathrm{C}$ allele & 305 & 66.02 & 201 & 64.01 & 27.5 & & 1.0 (Ref.) & - \\
\hline & A allele & 157 & 33.98 & 113 & 35.99 & 27.2 & 0.21 & $1.33(0.86-2.08)$ & 0.19 \\
\hline
\end{tabular}

Patients carrying the rs11615 TT genotype (22.8 months) and the T allele (24.2 months) showed a significantly shorter median survival time when compared to patients with the GG genotype (33.7 months). The log-rank test showed significantly different survival time between the rs 11615 polymorphisms (Table 3). According to Cox proportional hazard regression analysis, the adjusted hazard ratios (HR) of OS in those carrying the rs11615 TT genotype and the T allele were 2.79 (1.15-7.26) and 1.84 (1.19-2.87) when using the wild-type genotype as a reference variable. However, we did not observe a significant association between the rs3212986 polymorphism and OS in gastric cancer patients.

\section{DISCUSSION}

Our study found that subjects carrying ERCC1 rs11615 TT showed a significantly poorer response to chemotherapy and shorter median survival time when compared with the GG genotype. Our data indicate that the ERCC1 rs 11615 polymorphism is a useful biomarker for assessing the response to chemotherapy and prognosis of gastric cancer, and our results may be valuable for designing individualizing patient treatment.

Polymorphisms in ERCC1 genes are reported to be a potential predictor for the response to chemotherapy and clinical outcome of cancer patients, such nasopharyngeal carcinoma, colorectal cancer, and non-small cell lung as well as ovarian cancer (Chen et al., 2013; Moxley et al., 2013; Oguri et al., 2013; Tiseo et al., 2013). For gastric cancer, 2 systemic reviews indicated that ERCC1 $\mathrm{rs} 11615 \mathrm{C}>\mathrm{T}$ polymorphisms are useful prognostic factors in gastric cancer treated with platinum-based chemotherapy (Liu et al., 2011; Wang et al., 2012). 
The results of our study agree with those of previous studies.

ERCC1 maps to chromosome 19q13.32, which consists of 10 exons and encoding a 297 -acetaldehyde ammonia product. It encodes a subunit for the NER pathway and is involved in repairing the interstrand and intrastrand cross-links induced by platinum/5-FU-based chemotherapy (Gustavsson et al., 2009; Liang et al., 2010; Wang et al., 2012). Because ERCC1 plays an important role in larger lesions covering 20-25 nucleotides, the DNA repair capacity of ERCC1 was considered to be a critical mechanism of resistance to platinum/5-FU-based drugs and radiation (Reed, 1998; Wood et al., 2001; Goode et al., 2002). Therefore, variants of ERCC1 play a role in the susceptibility to cancer. Previous studies indicated that variants of ERCC1 rs11615 have an impact on the clinical outcome of gastric patients receiving fluorouracil-based chemotherapy (Liang et al., 2010; Kim et al., 2011; Sonnenblick et al., 2012). Liang et al. (2010) reported that genetic polymorphisms of ERCC1-118C/C were associated with longer overall survival time of advanced gastric cancer patients treated with 5-FU-based combination chemotherapy. Another study conducted in Korea reported that ERCC1 expression was associated with tumor size, disease-free survival, and overall survival (Kim et al., 2011). However, the results conflict with those of 2 other studies (Yun et al., 2010; Wang et al., 2012). A study conducted in China has indicated that polymorphisms of ERCC1 are not associated with the response rate of 5-FU-based chemotherapy (Wang et al., 2012). Another study in Korea indicated that ERCC1 protein expression is not associated with the prognosis of gastric cancer (Yun et al., 2010).

There were several limitations to our study. First, cases were selected in one hospital. Therefore, selection bias could not be avoided. Second, some factors could have influenced the prognosis of gastric cancer, such as clinical status, other genetic factors, and ethnicities. However, these factors were not included in our analysis. Therefore, large multi-center studies including different ethnicities are warranted to further investigate the impact of ERCC1 polymorphisms on the prognosis of gastric cancer.

In conclusion, we reported that ERCC1 rs11615 TT can be used as a prognostic marker for the clinical outcome of gastric cancer patients treated with 5-FU-based chemotherapy. This suggests that detection of ERCC1 rs11615 may provide meaningful clinical information for individualized choices of 5-FU-based compound for treating gastric cancer. Further multicenter studies that include various populations are required to confirm the results of our study.

\section{ACKNOWLEDGMENTS}

The authors would like to thank the Affiliated Hospital of Yanbian University for providing help as well as patients who provided blood samples for our study.

\section{REFERENCES}

Chen C, Wang F, Wang Z, Li C, et al. (2013). Polymorphisms in ERCC1 C8092A predict progression-free survival in metastatic/recurrent nasopharyngeal carcinoma treated with cisplatin-based chemotherapy. Cancer Chemother. Pharmacol. 72: 315-322.

Ferlay J, Shin HR, Bray F, Forman D, et al. (2010). Estimates of worldwide burden of cancer in 2008: GLOBOCAN 2008. Int. J. Cancer 127: 2893-2917.

Friedberg EC (2001). How nucleotide excision repair protects against cancer. Nat. Rev. Cancer 1: 22-33.

Goode EL, Ulrich CM and Potter JD (2002). Polymorphisms in DNA repair genes and associations with cancer risk. Cancer Epidemiol. Biomarkers Prev. 11: 1513-1530. 
Gustavsson B, Kaiser C, Carlsson G, Wettergren Y, et al. (2009). Molecular determinants of efficacy for 5-FU-based treatments in advanced colorectal cancer: mRNA expression for 18 chemotherapy-related genes. Int. J. Cancer 124: $1220-1226$.

Huang ZH, Hua D, Du X, Li LH, et al. (2008). ERCC1 polymorphism, expression and clinical outcome of oxaliplatinbased adjuvant chemotherapy in gastric cancer. World J. Gastroenterol. 14: 6401-6407.

Kim KH, Kwon HC, Oh SY, Kim SH, et al. (2011). Clinicopathologic significance of ERCC1, thymidylate synthase and glutathione S-transferase P1 expression for advanced gastric cancer patients receiving adjuvant 5-FU and cisplatin chemotherapy. Biomarkers 16: 74-82.

Liang J, Li QF, Yao RY, Lu HY, et al. (2010). Association between genetic polymorphisms of ERCC1, XRCC1, GSTP1 and survival of advanced gastric cancer patients treated with oxaliplatin/5-Fu-based chemotherapy. Zhonghua Zhong Liu Za Zhi 32: 515-519.

Liu YP, Ling Y, Zhang YP and Liu BR (2011). Predictive values of platinum-related gene polymorphisms in gastric cancer patients on oxaliplatin-based adjuvant chemotherapy. Zhonghua Yi Xue Za Zhi 91: 256-259.

MacDonald JS (2004). Clinical overview: adjuvant therapy of gastrointestinal cancer. Cancer Chemother. Pharmacol. 54 (Suppl 1): S4-S11.

Miller AB, Hoogstraten B, Staquet M and Winkler A (1981). Reporting results of cancer treatment. Cancer 47: 207-214.

Mlak R, Krawczyk P, Ramlau R, Kalinka-Warzocha E, et al. (2013). Predictive value of ERCC1 and RRM1 gene singlenucleotide polymorphisms for first-line platinum- and gemcitabine-based chemotherapy in non-small cell lung cancer patients. Oncol. Rep. 30: 2385-2398.

Moxley KM, Benbrook DM, Queimado L, Zuna RE, et al. (2013). The role of single nucleotide polymorphisms of the ERCC1 and MMS19 genes in predicting platinum-sensitivity, progression-free and overall survival in advanced epithelial ovarian cancer. Gynecol. Oncol. 130: 377-382.

Oguri T, Mitsuma A, Inada-Inoue M, Morita S, et al. (2013). Genetic polymorphisms associated with oxaliplatin-induced peripheral neurotoxicity in Japanese patients with colorectal cancer. Int. J. Clin. Pharmacol. Ther. 51: 475-481.

Reed E (1998). Platinum-DNA adduct, nucleotide excision repair and platinum based anti-cancer chemotherapy. Cancer Treat. Rev. 24: 331-344.

Rumiato E, Cavallin F, Boldrin E, Cagol M, et al. (2013). ERCC1 C8092A (rs3212986) polymorphism as a predictive marker in esophageal cancer patients treated with cisplatin/5-FU-based neoadjuvant therapy. Pharmacogenet. Genomics 23: 597-604.

Sonnenblick A, Rottenberg Y, Kadouri L, Wygoda M, et al. (2012). Long-term outcome of continuous 5-fluorouracil/ cisplatin-based chemotherapy followed by chemoradiation in patients with resected gastric cancer. Med. Oncol. 29: 3035-3038.

Tiseo M, Bordi P, Bortesi B, Boni L, et al. (2013). ERCC1/BRCA1 expression and gene polymorphisms as prognostic and predictive factors in advanced NSCLC treated with or without cisplatin. Br. J. Cancer 108: 1695-1703.

Volker M, Moné MJ, Karmakar P, van Hoffen A, et al. (2001). Sequential assembly of the nucleotide excision repair factors in vivo. Mol. Cell 8: 213-224.

Wang Z, Chen JQ, Liu JL, Qin XG, et al. (2012). Polymorphisms in ERCC1, GSTs, TS and MTHFR predict clinical outcomes of gastric cancer patients treated with platinum/5-Fu-based chemotherapy: a systematic review. $B M C$ Gastroenterol. 12: 137.

Wöhrer SS, Raderer M and Hejna M (2004). Palliative chemotherapy for advanced gastric cancer. Ann. Oncol. 15: 15851595.

Wood RD, Mitchell M, Sgouros J and Lindahl T (2001). Human DNA repair genes. Science 291: 1284-1289.

Yang Z, Fang X, Pei X and Li H (2013). Polymorphisms in the ERCC1 and XPF genes and risk of breast cancer in a Chinese population. Genet. Test. Mol. Biomarkers 17: 700-706.

Yin M, Yan J, Martinez-Balibrea E, Graziano F, et al. (2011). ERCC1 and ERCC2 polymorphisms predict clinical outcomes of oxaliplatin-based chemotherapies in gastric and colorectal cancer: a systemic review and meta-analysis. Clin. Cancer Res. 17: 1632-1640.

Yun J, Kim KM, Kim ST, Kim JH, et al. (2010). Predictive value of the ERCC1 expression for treatment response and survival in advanced gastric cancer patients receiving cisplatin-based first-line chemotherapy. Cancer Res. Treat. 42: 101-106.

Zamble DB, Mu D, Reardon JT, Sancar A, et al. (1996). Repair of cisplatin - DNA adducts by the mammalian excision nuclease. Biochemistry 35: 10004-10013. 